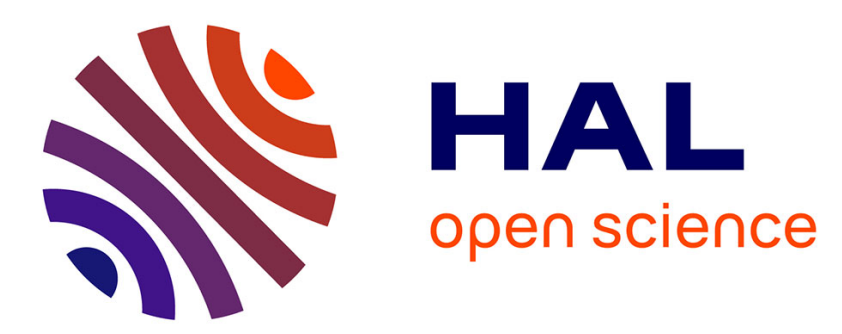

\title{
Acceleration Feedback via an algebraic state estimation method
}

\author{
Romain Delpoux, Hebertt Sira-Ramirez, Thierry Floquet
}

\section{To cite this version:}

Romain Delpoux, Hebertt Sira-Ramirez, Thierry Floquet. Acceleration Feedback via an algebraic state estimation method. 52th IEEE Conference on Decision and Control,, Dec 2013, Florence, Italy. hal-00881164

\section{HAL Id: hal-00881164 https://hal.inria.fr/hal-00881164}

Submitted on 7 Nov 2013

HAL is a multi-disciplinary open access archive for the deposit and dissemination of scientific research documents, whether they are published or not. The documents may come from teaching and research institutions in France or abroad, or from public or private research centers.
L'archive ouverte pluridisciplinaire HAL, est destinée au dépôt et à la diffusion de documents scientifiques de niveau recherche, publiés ou non, émanant des établissements d'enseignement et de recherche français ou étrangers, des laboratoires publics ou privés. 


\title{
Acceleration Feedback via an algebraic state estimation method
}

\author{
Romain Delpoux, Hebertt Sira-Ramírez and Thierry Floquet
}

\begin{abstract}
In many mechanical systems, only accelerations are available for feedback purposes. For example, certain aerospace, positioning systems and force-position controllers in robotic systems, use accelerometers as the only sensing device. This paper presents initial steps towards an algebraic approach for the state estimation based feedback control problem in systems where the highest order derivative of the controlled variable is available. An illustrative case is presented dealing with the trajectory tracking problem for a second order position system on which only the acceleration is available for measurement. Based on an algebraic approach, an on-line algebraic estimator is developed for the unmeasured position and velocity variables. The obtained expressions depend solely on iterated integrals of the measured acceleration output and of the control input. The approach is robust to noisy measurement and it has the advantage to provide fast, on-line, non-asymptotic state estimations in the form of formulae requiring only the input and the output of the system. Based on these estimations, a linear feedback control law including estimated position error integrals is designed illustrating the possibilities of acceleration feedback via algebraic state estimation.
\end{abstract}

Index Terms-Algebraic state estimation, acceleration feedback, linear time invariant system.

\section{INTRODUCTION}

This article is concerned with preliminary results on an algebraic approach to the feedback control of systems where only the highest order time derivative of the output is available for feedback purposes. The possibilities of the algebraic approach are illustrated by considering the position reference trajectory tracking problem for a sufficiently simple second order system in which only the acceleration variable is measured. In many mechanical systems, this corresponds to the classical acceleration feedback problem. This problem has practical importance since, in many systems, such as aerospace systems, only the use of accelerometers as sensing devices is available.

Acceleration feedback is of common practice in vibration control schemes, attenuating unbalanced responses, in rotordynamics problems (see Inman [12]). It is also extensively used in impact related problems in force position control of robotic systems (see Wu et al. [22]). Acceleration feedback has also been proposed for the feedback control of plates in seismically excited buildings (see Jabbari et al. [13])

The algebraic approach to state and parameter estimation was introduced by M. Fliess and H. Sira-Ramírez in [8],

R. Delpoux and T. Floquet are with Laboratoire d'Automatique, Génie Informatique et Signal (CNRS UMR 8219), Ecole Centrale de Lille and Université Lille I, 59651 Villeneuve d'Ascq cedex, France and Team Non-A, INRIA Lille Nord Europe, France romain. delpouxdec-lille.fr and thierry.floquet@ec-lille.fr

H. Sira-Ramírez is with the Seccion de Mecatronica, Centro de Investigación y Estudios Avanzados del IPN (Cinvestav-IPN), 07300 Mexico D.F., Mexico hsira@cinvestav.mx
[9]. This method has already been successfully applied to parameter estimation [2], [15], [16], [18], [19], to abrupt change detections and the efficient identification of time delays [1], [5], [21]. Numerical differentiation of noisy signals may also benefit from this approach, as demonstrated in [17], [20].

In this note, the reference trajectory tracking problem is considered for a second order position system in which only the acceleration variable is measured for feedback purposes. Algebraic manipulations are performed in order to on-line extract relevant position and velocity information for feedback purposes. The estimator is non asymptotic in nature, and it allows the simultaneous estimation of the unmeasured states of the system, the state initial conditions and the integral of the position variable. The estimated signals are readily used to devise a full state feedback law including integral error compensation. Unlike traditional estimation methods, the proposed estimator is non-asymptotic in nature. The estimated signals are obtained, after a very small time interval, via exact algebraic formulae, allowing finite time estimates of the variables required for feedback. In the case treated, these variables are immediately used in conforming a PID feedback controller for reference position trajectory tracking tasks. The estimated quantities are expressed as a function of integrals of the measured output and of the control input to the system. Although the approach does not need any statistical knowledge of the measurement and plant noises, analysis of the stochastic kind may be easily incorporated In more general cases, differentiation of control inputs may be necessary. We remark that in those cases any suitable differentiator could be used for completing the estimation based feedback control scheme. A classical approach to signal differentiation is based on least-squares polynomial fitting, or interpolation (see i.e. [4], [11]) in offline applications. Differentiation methods based on observer design may be found in the control literature (see [10] for a linear approach). Non-linear methods, such as sliding modes differentiator [14], have also gained popularity in recent times. However, all these observer based signal differentiation methods may be highly sensitive to measurement noises. In the algebraic method here proposed only integration of the measured outputs are required, thus providing a convenient low pass filtering action on the available signals.

This article consists of three parts. The first section presents the problem statement. In the second section, the algebraic estimator is developed. Finally, the last section is devoted to the numerical results. The conclusions are presented at the end of the article. 


\section{PROBLEM STATEMENT}

Consider a second order linear scalar system with dynamic input/output relationship given by:

$$
\begin{aligned}
\ddot{x}(t) & =a \dot{x}(t)+b x(t)+u(t), \\
y(t) & =\ddot{x}(t)
\end{aligned}
$$

with $x(t) \in \mathbb{R}$, labeled as the position variable, the parameters $a$ and $b$ are assumed to be known constants and $u(t) \in \mathbb{R}$ is the control input. The output $y(t)$ of the system coincides with the second order time derivative of $x(t)$, i.e., it represents the acceleration.

Provided the coefficient $b$ satisfies: $b \neq 0$, then the system (1) is algebraically observable (see Diop and Fliess [3]). Indeed, the state variables of the system: $x(t), \dot{x}(t)$, can be uniquely expressed as functions of the input $u(t)$, the output $y(t)$, and a finite number of time derivatives of these two variables. One easily obtains,

$$
\begin{aligned}
\dot{x}(t)= & b^{-1}[\dot{y}(t)-a y(t)-\dot{u}(t)] \\
x(t)= & b^{-1}\left[-a b^{-1} \dot{y}(t)+\left(1+a^{2} b^{-1}\right) y(t)\right. \\
& \left.+a b^{-1} \dot{u}(t)-u(t)\right] .
\end{aligned}
$$

The system is also trivially flat (see Fliess et al. [6]), with unmeasured, but observable, flat output $x(t)$.

The control objective is to design an output feedback control law for the accurate tracking of a reference position $x(t)$, denoted by $x^{*}(t)$. For this purpose, differentiators have been used in the past to estimate the states of the system from (2). This solution would give satisfactory results in the absence of noise measurement but, as it turns out, the scheme is quite sensitive to those uncertainties and it exhibits a lack of robustness. An alternative approach, presented here, does not require any differentiations. The measurement noises, which will be assumed to be zero mean just for simplicity, undergo iterated integrations and, thus, they are conveniently attenuated.

\section{ALGEBRAIC STATE ESTIMATION}

The procedure for the algebraic estimation of the states of system (1) entitles the construction of an independent set of, time-varying, linear equations with coefficients given by suitable iterated integrals (possibly in time-convolution form) of inputs and outputs. One seeks that the state variables to be estimated, i.e., $x(t)$ and $\dot{x}(t)$, conform a subset of the set of all the required unknowns arising from the involved algebraic manipulations. In our case, the generated set of unknowns turn out to include, aside from $x(t)$ and $\dot{x}(t)$, the initial conditions: $x(0), \dot{x}(0)$ and, quite conveniently for control purposes, the integral of the unknown position variable $x(t)$, i.e., $\int_{0}^{t} x(\tau) d \tau$.

In order to generate a sufficient number of independent linear equations, the use the algebraic derivative method, introduced in Fliess and Sira-Ramírez [8], proves to be highly convenient. In the frequency domain, this procedure consists of differentiating with respect to the complex variable $s$, as many times as required, the Laplace transformed expression of either the system equation or of a significantly related equation (in our particular case, this entitles the plant equation and the output equation in (1). The aim is first to eliminate the constant initial conditions that may appear due to Laplace transformations of time derivatives. If more equations are necessary one may allow the explicit presence of initial conditions considering them as additional unknowns by differentiating a smaller amount of times with respect to $s$. Once this is accomplished, one proceeds to multiplying the resulting expressions by suitable powers of $s^{-1}$, so as to eliminate all strictly positive powers of $s$ (which in the time domain would represent time differentiations) and obtain only integrations in the time domain. The algebraic derivative procedure has a counterpart entirely applicable in the time domain, where the iterated algebraic differentiations are replaced by direct multiplication by positive powers of the time variable $-t$. The multiplication by negative powers of $s$ in the frequency domain is replaced by iterated integrations in the time domain. The procedure in the time domain also advantageously uses integration by parts in further contributing to the elimination of time differentiations and initial conditions. The resulting expressions, are valid over any time interval $[0, t]$ irrespectively of how small this interval may be.

One starts by directly integrating the measured output $y(t)$ on the time interval $[0, t]$. One obtains a first linear equation involving the unknown velocity state $\dot{x}(t)$ and its initial value:

$$
\int_{0}^{t} y(\tau) d \tau=\int_{0}^{t} \ddot{x}(\tau) d \tau=\dot{x}(t)-\dot{x}(0) .
$$

Multiplying the measured output $y(t)$ by $t$ and integrating the resulting expression, one obtains a second linear equation after integration by parts:

$$
\begin{aligned}
\int_{0}^{t} \tau y(\tau) d \tau=\int_{0}^{t} \tau \ddot{x}(\tau) d \tau & =t \dot{x}(t)-\int_{0}^{t} \dot{x}(\tau) d \tau \\
& =t \dot{x}(t)-x(t)+x(0) .
\end{aligned}
$$

A third linear equation is obtained by multiplying the measured output by the factor $t^{2}$ and integrating it just once. One in led to the following expression after integration by parts:

$$
\begin{aligned}
\int_{0}^{t} \tau^{2} y(\tau) d \tau & =\int_{0}^{t} \tau^{2} \ddot{x}(\tau) d \tau \\
& =t^{2} \dot{x}(t)-2 \int_{0}^{t} \tau \dot{x}(\tau) d \tau \\
& =t^{2} \dot{x}(t)-2 t x(t)+2 \int_{0}^{t} x(\tau) d \tau .
\end{aligned}
$$

A fourth linear equation is obtained as follows: differentiating, with respect to time, both sides of the plant equation, (1), one obtains:

$$
\dot{y}(t)=a y(t)+b \dot{x}(t)+\dot{u}(t) .
$$

Multiplying now both sides of this last expression by $t$ followed by integration by parts over the time interval $[0, t]$ 
implies:

$$
\begin{aligned}
t y(t)- & \int_{0}^{t} y(\tau) d \tau-a \int_{0}^{t} \tau y(\tau) d \tau \\
-t u(t) & +\int_{0}^{t} u(\tau) d \tau \\
& =b t x(t)-b \int_{0}^{t} x(\tau) d \tau
\end{aligned}
$$

The last equation is the plant equation itself, written now, as follows,

$$
y(t)=a \dot{x}(t)+b x(t)+u(t) .
$$

Thus, from equations (1), (3), (4) and (5), and (7), one ends up with five independent equations in five unknown variables, lumped in the vector $\gamma(t)$ defined as:

$$
\gamma(t)=\left(\int_{0}^{t} x(\tau) d \tau, x(t), \dot{x}(t), x(0), \dot{x}(0)\right)^{T} .
$$

In other words, one has a set of linear equations, represented by:

$$
P(t) \gamma(t)=q(t)
$$

where:

$$
P(t)=\left[\begin{array}{ccccc}
0 & b & a & 0 & 0 \\
0 & 0 & 1 & 0 & -1 \\
0 & -1 & t & 1 & 0 \\
2 & -2 t & t^{2} & 0 & 0 \\
-b & b t & 0 & 0 & 0
\end{array}\right]
$$

and:

$$
q(t)=\left[\begin{array}{c}
y(t)-u(t) \\
\int_{0}^{t} y(\tau) d \tau \\
\int_{0}^{t} \tau y(\tau) d \tau \\
\int_{0}^{t} \tau^{2} y(\tau) d \tau \\
F(t)
\end{array}\right],
$$

where

$$
\begin{gathered}
F(t)=\quad t y(\tau)-\int_{0}^{t} y(\tau) d \tau-a \int_{0}^{t} \tau y(\tau) d \tau \\
-t u(t)+\int_{0}^{t} u(\tau) d \tau
\end{gathered}
$$

which depends on time $t$, and integral expressions involving only the measured output, $y(t)$, and the known control input, $u(t)$. The vector $\gamma(t)$ is conformed by a superset of the set of state variables to be estimated.

Notice that the matrix $P(t)$ is singular at time $t=0$. However, this matrix is indeed invertible for any time $t \geq$ $\epsilon>0$. the solution for $\gamma(t)$, is simply obtained by:

$$
\gamma(t)=P(t)^{-1} q(t), \quad \forall t \geq \epsilon>0 .
$$

Remark 1: Note that $\operatorname{det}(P(t))=-b^{2} t^{2}$ which means that $P(t)$ is invertible for any strictly positive time $t$ provided the constant parameter, $b$, is non-zero. This makes perfect sense since the system is not observable whenever $b=0$.
As in the algebraic method for parameter identification, before time $t=\epsilon>0$, the estimated states are left to be undetermined. Therefore, the formula for the estimation of the states (11) may be used, not from time $t=0$, but from a slightly later time, $t=\epsilon$. The design parameter $\epsilon$ being small and strictly positive. Moreover if $t \rightarrow \infty$ then $\|P(t)\| \rightarrow \infty$ also while this is not necessary the case for $\|q(t)\|$. The time $t$ must also be upper bounded. This can be easily solved by a time reset after a time $t=t_{\max }$.

Thus, $\hat{\gamma}(t)$ is estimated as follows:

$$
\hat{\gamma}(t)= \begin{cases}\text { arbitrary } & \text { for } t \in[0, \epsilon[ \\ P(t)^{-1} q(t) & \text { for } t \in\left[\epsilon, t_{\max }\right] .\end{cases}
$$

Remark 2: Notice that, even in the low dimensional case at hand, there is quite some freedom to generate the required set of independent algebraic linear equations in the five unknowns constituting the vector $\gamma(t)$. For a systematic round down, one may start by first integrating, once, the output equation $y(t)=\ddot{x}(t)$. Then, proceed to integrate the same relation, twice. One, thus, obtains two independent equations. Then, multiplying the same output equation by $t$ and integrating yields, as explained above, one more equation. One may, then, multiply the output equation by $t^{2}$ and integrate it obtaining yet a fourth equation while having generated, so far, five unknowns. So far the plant equation has not been touched. This may be handled to additionally generate one more needed equation. One possibility is to consider, without integrations, the plant equation written as: $y(t)=a \dot{x}(t)+b x(t)+u(t)$, which is certainly independent of the previously generated. Alternatively, one may also integrate such an equation directly, or to multiply it by $t$ and integrate. One may also differentiate it first, multiply it by $t$ and integrate it as it was done above. In summary, one must algebraically generate an independent set of linear equations without letting the number of unknowns explode unnecessarily.

\section{NUMERICAL SIMULATIONS RESULTS}

Consider a second order mechanical system consisting of a spring and a damper attached to a mass which moves along the direction of the applied force on a frictionless surface. The dynamics of the system, in which the acceleration represents the output variable, is given by the set of equations:

$$
\begin{aligned}
m \ddot{x}(t)+c \dot{x}(t)+k x(t) & =u(t), \\
y(t) & =\ddot{x}(t),
\end{aligned}
$$

where the mass $m$ is taken to be $m=1[\mathrm{~kg}]$, the viscous friction coefficient is $c=2\left[\mathrm{~N} . \mathrm{s} . \mathrm{m}^{-1}\right]$ and the spring constant $k=4\left[\mathrm{~N} . \mathrm{m}^{-1}\right], x$ is the position variable, and the measured output is $y(t)$.

A feedback controller design is readily obtained for the reference position trajectory tracking task. Denote the desired position of the mass by $x^{*}(t)$. If no perturbations are present, then a classical PID controller plus suitable precompensation may be devised. If, however, the system were subject to classical additive perturbation inputs then, in general, a Generalized Proportional Integral (GPI) controller plus pre-compensation could be used for the trajectory tracking 
task (see [7] for revenant theoretical basis and [18] for some application of this type of controller). Consider, then, that no external perturbations are present and devise the following PID controller:

$$
\begin{aligned}
\frac{1}{m} u(t)= & \ddot{x}^{*}(t)-\left(k_{2}-\frac{c}{m}\right)\left(\dot{x}(t)-\dot{x}^{*}(t)\right) \\
& -\left(k_{1}-\frac{k}{m}\right)\left(x(t)-x^{*}(t)\right) \\
& -k_{0} \int_{0}^{t}\left(x(\tau)-x^{*}(\tau)\right) d \tau,
\end{aligned}
$$

which can be realized whenever the system parameters and the state variables $x(t), \dot{x}(t)$, are known. Choosing appropriate design parameter $k_{2}$ and $k_{1}, k_{0}$, the controller guarantees the exponentially asymptotically stability of the position reference trajectory tracking error, $e(t)=x(t)-x^{*}(t)$, in the closed-loop dynamics :

$$
\ddot{e}(t)+k_{2} \dot{e}(t)+k_{1} e(t)+k_{0} \int_{0}^{t} e(\tau) d \tau .
$$

The set of design parameters is chosen so as to render the closed-loop characteristic polynomial,

$$
p(s)=s^{3}+k_{2} s^{2}+k_{1} s+k_{0},
$$

into a Hurwitz polynomial with desirable stable roots in the left half of the complex plane. The polynomial (15), can be identified to the general third order characteristic polynomial,

$$
\begin{aligned}
p_{d}(s) & =\left(s^{2}+2 \zeta \omega_{n} s+\omega_{n}^{2}\right)(s+p) \\
& =s^{3}+\left(p+2 \zeta \omega_{n}\right) s^{2}+\left(\omega_{n}^{2}+2 \zeta \omega_{n} p\right) s+\omega_{n}^{2} p,
\end{aligned}
$$

with $\zeta$ being the desired damping coefficient and $\omega_{n}$ the desired natural frequency of the dominant second order closed loop dynamics.

The proposed controller (13) obviously requires the position and the velocity variables, $x(t)$ and $\dot{x}(t)$ which are not available since only the acceleration $\ddot{x}(t)$ is measured. Using the algebraic estimation procedure described above in (11) we can, however, readily estimate the unknown states to realize the control.

A desired smooth reference trajectory for the position $x(t)$, is specified during a time interval of the form: $\left[t_{i}, t_{f}\right]$, starting from a initial position value $x^{*}\left(t_{i}\right)$ and ending at a final position value $x^{*}\left(t_{f}\right)$. The smooth trajectory is designed so that the following eight constraints are satisfied on the initial and final positions: $x^{*}\left(t_{i}\right)=0, x^{*}\left(t_{f}\right)=1, \dot{x}^{*}\left(t_{i}\right)=0$, $\dot{x}^{*}\left(t_{f}\right)=0, \ddot{x}^{*}\left(t_{i}\right)=0$ and $\left.\ddot{x}^{*}\left(t_{f}\right)=0\right)$. Thus a suitable Bèzier polynomial could be of degree 7 :

$$
\begin{aligned}
x^{*}(t)= & x^{*}\left(t_{i}\right)+\left(x^{*}\left(t_{f}\right)-x^{*}\left(t_{i}\right)\right)\left(a_{0} \Delta(t)^{7}\right. \\
& +a_{1} \Delta(t)^{6}+a_{2} \Delta(t)^{5}+a_{3} \Delta(t)^{4} \\
& +a_{4} \Delta(t)^{3}+a_{5} \Delta(t)^{2}+a_{6} \Delta(t)+a_{7},
\end{aligned}
$$

where

$$
\Delta(t)=\frac{t-t_{i}}{t_{f}-t_{i}}
$$

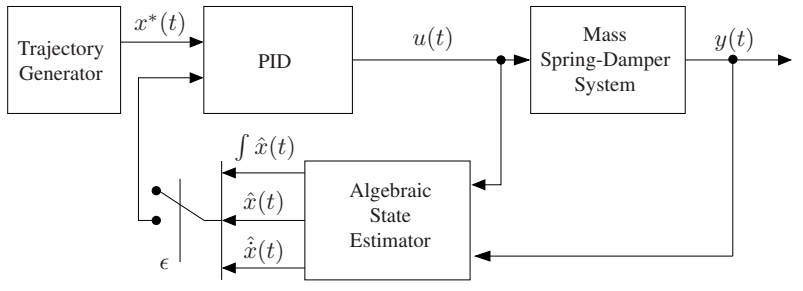

Fig. 1. Feedback control via output acceleration feedback and algebraic state estimation.

and

$$
\begin{aligned}
& a_{0}=20, \quad a_{1}=-70, \quad a_{2}=84, \quad a_{3}=-35, \\
& a_{4}=0, \quad a_{5}=0, \quad a_{6}=0, \quad a_{7}=0 .
\end{aligned}
$$

Simulations were carried out using the Matlab/Simulink package. The trajectory tracking objective consists in driving the mass from an arbitrary initial position $x^{*}\left(t_{i}\right)=0[\mathrm{~m}]$ to a final position $x^{*}\left(t_{i}\right)=1[\mathrm{~m}]$. In order to illustrate the accuracy of the initial condition estimation performed by the proposed algebraic estimator, the initial conditions of the system were chosen to be different from zero: $x(0)=0.1[\mathrm{~m}]$ and $\dot{x}(0)=-0.5\left[m_{. s}{ }^{-1}\right]$. The PID controller gains: $k_{2}$ and $k_{1}$ and $k_{0}$ are chosen in correspondence with $\zeta=\frac{1}{\sqrt{2}}, \omega_{n}=$ $20\left[\right.$ rad.s $\left.s^{-1}\right]$ and $p=\zeta \omega_{n}$. It is important to point out that the algebraic estimator does not require any traditional parameter tuning. The only parameter to be set is the parameter $\epsilon$, which represents the instant of time after which accurate estimations become available. We have chosen this parameter (for the noiseless case) to coincide with the length of the sampling interval which, in the simulations was taken to be $\epsilon=10^{-4}[s]$. The controlled system is depicted Figure 1. Generally speaking, when noise processes are present in the plant or in the measurements, the parameter $\epsilon$ should be chosen substantially larger in order to allow for a better signal-to-noise ratio in the right hand side of 11 .

\section{A. Estimation in the absence of measurement noise}

The simulation results corresponding to the first case (no measurement noise) are shown in Figure 2. Plot (IV) depicts the position trajectory response, $x(t)$, along with the desired reference trajectory $x^{*}(t)$ and the estimated position. Plot (V) contains the position estimation error, which is zero for $t \geq \epsilon$. The third plot (VI) depicts the trajectory tracking error for the position variable. As mentioned in the introduction, the algebraic estimation leads to very fast, online estimation. Similar plots are presented for the integral of the position variables (plot (I), (II) and (III)), and for the velocity variables (plot (VII), (VIII) and (IX)) The graph in plot $(\mathrm{X})$ shows the measured output, i.e., the acceleration. The initial conditions, $x(0)$ and $\dot{x}(0)$, are perfectly estimated, as shown in plots (XI) and (XII).

\section{B. Estimation with measurement noise}

Simulations were also carried out to assess the performance of the output acceleration feedback scheme when a measurement noise process is present in the acceleration measurement. A computer generated stochastic noise process 

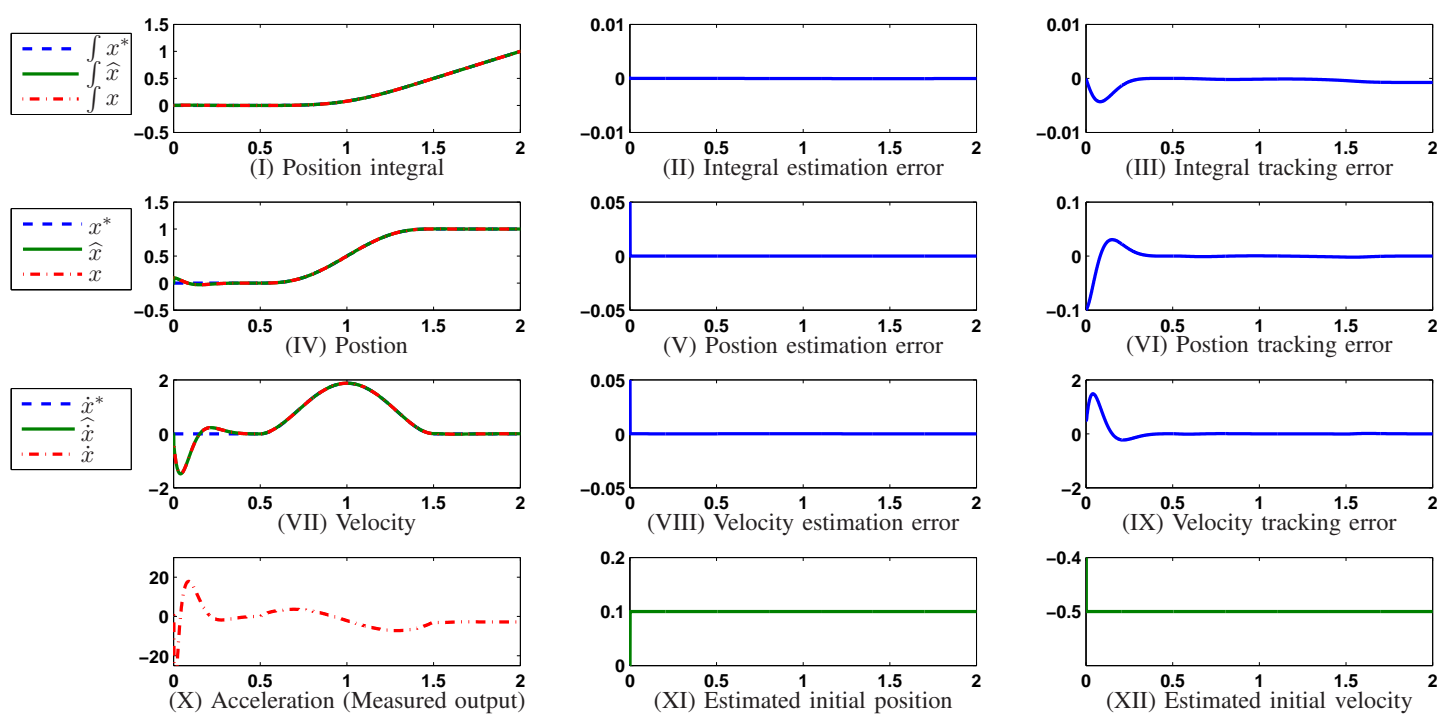

Fig. 2. Simulation of the algebraic estimation based output acceleration feedback controller. No output measurement noise.

was added to the measured acceleration. This process was realized as a sequence, with one element at each sampling time, of zero mean Gaussian distributed random. The variance is adjusted in such a way that the signal to noise ratio in $\mathrm{dB}$, i.e., $S N R=10 \log _{10}\left(\frac{\sum\left|y_{i}\right|^{2}}{\sum\left|\varpi_{i}\right|^{2}}\right)$, $\varpi$ being the added noise, corresponds to $S N R=25 \mathrm{~dB}$ with $\epsilon$ equal to $0.05[s]$. The simulation results are shown in Figure 3, in correspondence with those shown in Figure 2. This set of figures shows that even in the presence of noise the trajectory tracking is still satisfactorily accomplished. The noise effect is present at the beginning of the simulations. However, due the the fact the the inputs and the outputs are being integrated, these noise effects are rapidly attenuated. After 0.5 seconds, the noise has already been considerably reduced. It should nevertheless be noted that the noise has a little more influence on the estimation of the initial conditions. Nevertheless, the initial conditions are still estimated in a quite precise fashion.

\section{CONCLUSIONS AND FUTURE WORKS}

Controlling a second order system from acceleration measurements alone constitutes an important task in some practical applications of automatic control. Indeed, in many mechanical systems, accelerometers are the only available sensing devices for feedback control. This is particularly so in the control of some unmanned vehicles used in aerospace and also in some positioning control applications.

In this article, we have proposed a novel approach for feedback control of second order systems based on the availability of the acceleration signal alone. The approach uses model-based algebraic state estimation in a novel fashion. An illustrative example has been presented based on a simple second order position mechanical system on which only the acceleration variable is available for measurement. The velocity and the position variables are obtainable in a nonasymptotic fashion, i.e., in an almost instantaneous fashion, from: the acceleration output signal, the control input signal and iterated (convolution) integrals of these two variables. No tuning of gains is required for the proposed linear, fast, state estimator. Only a time reset is needed to overcome the sensitivity problems which may be caused by the integration of the time variable. As a bonus, the initial conditions of the plant state and the integral of the position variable are also obtained, substantially easing the implementation of integral position action in the control loop. The proposed approach has definite noise processing advantages over schemes based on dynamical differentiations and it is substantially faster than those methods based on passivity or energy dissipation results.

The results of this article have been illustrated via numerical simulations on a second order mass-spring-dumper system in a position reference trajectory tracking task. A linear feedback control of the PID type was readily designed for the feedback purposes on the basis of the obtained on-line estimates. The simulations have shown excellent results, even in the presence of measurement noise. We have confirmed that the estimation is quite fast and it occurs in a, small, finite amount of time. When measurement noise is added, the simulations have shown that the noise was also quickly attenuated by the integration structure present in the estimator. Finally, using these estimations, the designed control law exhibits an excellent performance and output tracking qualities. The tracking error is reasonably small even in the presence of measurement noises.

The proposed results might be generalized with some additional efforts. It would be interesting, however, to apply this algorithm to a larger class of systems. Generally speaking, algebraic methods for state estimation can be similarly ap- 

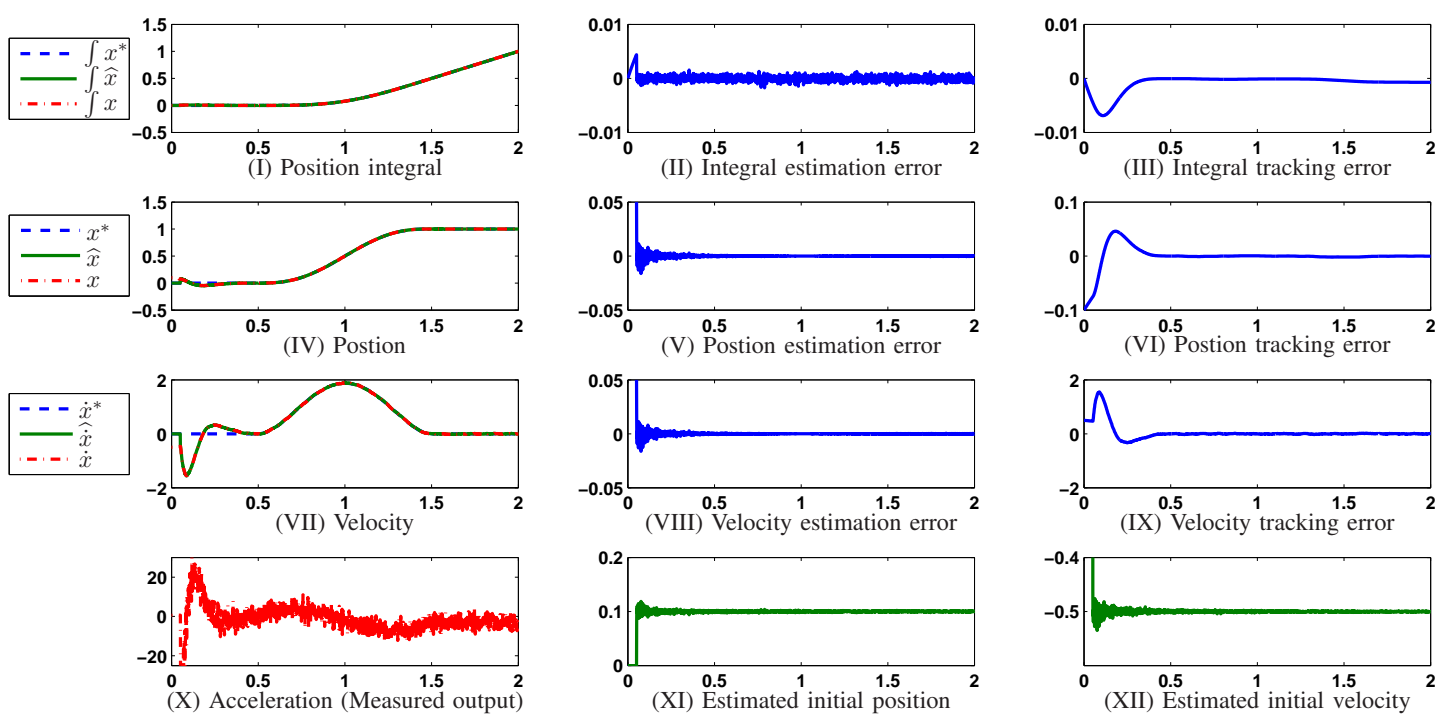

Fig. 3. Simulation of the algebraic estimation based output acceleration feedback controller. Measurement output noise included.

plied to the case of additively perturbed systems. Particularly, in the case of classical additive plant perturbation inputs (steps, ramps, etc.), some previous algebraic manipulations are required in order to annihilate the undesired effects of such perturbations.

\section{ACKNOWLEDGMENTS}

This work was supported by Nord-Pas de Calais Regional Council and FEDER through the Contrat de Projets Etat Region (CPER) 2007-2013.

\section{REFERENCES}

[1] L. Belkoura, J.-P. Richard, and M. Fliess. Parameters estimation of systems with delayed and structured entries. Automatica, 45(5):1117$1125,2009$.

[2] R. Delpoux and T. Floquet. On-line parameter estimation of a magnetic bearing. In 19th Mediterranean Conference on Control Automation, pages 388-393, Corfu, Greece, 2011.

[3] S. Diop and M. Fliess. Nonlinear observability, identifiability and persistent trajectories. In 36th IEEE Conference on Decision and Control, pages 714-719, Brighton, England, 1991.

[4] T.-E. Duncan, P. Mandl, and B. Pasik-Duncan. Numerical differentiation and parameter estimation in higher-order linear stochastic systems. IEEE Transactions on Automatic Control, 41(4):522-532, 1996.

[5] M. Fliess, C. Join, and M. Mboup. Algebraic change-point detection. Applicable Algebra in Engineering, Communication and Computing, 21:131-143, 2010

[6] M. Fliess, J. Lévine, and P. Rouchon. Flatness and defect of nonlinear systems: Introductory theory and examples. International Journal of Control, 61:1327-1361, 1995.

[7] M. Fliess, M. Mboup, H. Mounier, and H. Sira-Ramírez. Correcteurs proportionnels-intégraux généralisés. ESAIM: Control, Optimisation and Calculus of Variations, 9:151-168, 2003.

[8] M. Fliess and H. Sira-Ramírez. An algebraic framework for linear identification. ESAIM: Control, Optimisation and Calculus of Variations, 9:151-168, January 2003.

[9] M. Fliess and H. Sira-Ramírez. Closed-loop parametric identification for continuous-time linear systems via new algebraic techniques. In H Garnier \& L Wang, editor, Identification of Continuous-time Models from Sampled Data, Advances in Industrial Control, pages 362-391. Springer, 2008.
[10] S. Ibrir. Linear time-derivative trackers. Automatica, 40(3):397-405, 2004.

[11] S. Ibrir and S. Diop. A numerical procedure for filtering and efficient high-order signal differentiation. International Journal of Applied Mathematics and Computer Science, 14(2):201-208, 2004.

[12] D.-J. Inman. Vibration with Control. Academic Press John Wiley \& Sons, Ltd., England, 2006.

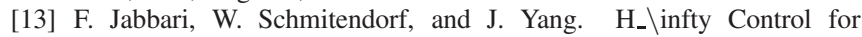
Seismic-Excited Buildings with Acceleration Feedback. Journal of Engineering Mechanics, 9:994-1002, 1995.

[14] A. Levant. Robust exact differentiation via sliding mode technique. Automatica, 34(3):379-384, March 1998.

[15] J. Linares-Flores, H. Sira-Ramírez, E. Yescas-Mendoza, and J.-J. Vásquez-Sanjuan. A comparison between the algebraic and the reduced order observer approaches for on-line load torque estimation in a unit power factor rectifier-DC motor system. Asian Journal of Control, 14(1):45-57, 2012.

[16] M. Mboup. Parameter estimation for signals described by differential equations. Applicable Analysis: An International Journal, 88(1):2952, 2009.

[17] M. Mboup, C. Join, and M. Fliess. Numerical differentiation with annihilators in noisy environment. Numerical Algorithms, 50:439467, 2009.

[18] R. Morales, V. Feliu, and H. Sira-Ramírez. Nonlinear Control for Magnetic Levitation Systems Based on Fast Online Algebraic Identification of the Input Gain. IEEE Transactions on Control Systems Technology, 19(4):757-771, 2011.

[19] W. Perruquetti, V. Bonnet, M. Mboup, R. Ushirobira, and P. Fraisse. An algebraic approach for human posture estimation in the sagittal plane using accelerometer noisy signal. In 51st IEEE Conference on Decision and Control, pages 7389-7394, Maui, Hawaii, United States, December 2012. Ieee.

[20] S. Riachy, Y. Bachalany, M. Mboup, and J.-P. Richard. Différentiation numérique multivariable I : estimateurs algébriques et structure. In Gième Conférence Internationale Francophone d'Automatique, Nancy, France, 2010.

[21] Z. Tiganj, M. Mboup, C. Pouzat, and L. Belkoura. An algebraic method for eye blink artifacts detection in single channel EEG recordings. In 17th International Conference on Biomagnetism, volume 28, pages 175-178, Dubrovnik, Croatia, 2010.

[22] Y. Wu, T.-J. Tarn, N. Xi, and A. Isidori. On Robust Impact Control via Positive Acceleration Feedback for Robot Manipulators, 1996. 